\title{
SÍNDROME HELLP: EXPERIENCIA DEL HOSPITAL REGIONAL DE PUERTO MONTT, 2000-2006
}

\author{
José Caro M. ${ }^{1,2}$, Carlos Anwandter Sch. ${ }^{1,2}$, Soledad Schaffeld P. ${ }^{1}$, Fernando Vega \\ G. ${ }^{1}$, Carolina López $S .{ }^{a}$, Jacqueline Jara P. ${ }^{a}$ \\ 1 Unidad de Alto Riesgo Obstétrico, Servicio de Obstetricia y Ginecología, Hospital de Puerto Montt. ${ }^{2}$ Departamento de \\ Obstetricia y Ginecología, Escuela de Medicina, Universidad San Sebastián Puerto Montt.
}

a Internas de Medicina, Escuela de Medicina, Universidad San Sebastián, Puerto Montt.

\section{RESUMEN}

Objetivos: Caracterizar el síndrome HELLP en una población obstétrica de la décima Región de Chile. Método: Análisis de 33 pacientes con síndrome HELLP asistidas en el hospital de Puerto Montt, en el período 2000-2006. Resultados: La incidencia fue 1,3 casos por mil partos. La edad gestacional promedio de presentación fue de 33 semanas. En $84,8 \%$ de las pacientes el diagnóstico fue anteparto. El $27 \%$ de las pacientes recibió dexametasona. La principal complicación materna fue la insuficiencia renal aguda. El $91 \%$ de las pacientes presentó hipertensión arterial. No hubo muertes maternas. El peso promedio de los recién nacidos fue 2.048 gramos; $42 \%$ con edad gestacional menor a 34 semanas; $34,4 \%$ pesó menos de 1500 gramos; $9,4 \%$ presentó depresión neonatal severa a los 5 minutos; $12,1 \%$ falleció en el período neonatal. Conclusiones: El síndrome HELLP es una patología de baja incidencia y con elevada morbimortalidad neonatal.

\section{PALABRAS CLAVE: Síndrome HELLP, pronóstico perinatal, dexametasona}

\section{SUMMARY}

Objectives: To characterize HELLP syndrome. Method: Analysis of 33 patients with HELLP syndrome assisted at the Puerto Montt hospital, in the period 2000-2006. Results: The incidence was 1.3 cases per thousand births. The average gestational age at presentation was 33 weeks; $84.4 \%$ of patient had prepartum diagnosis; $27 \%$ of patients received dexamethasone. The main complication was maternal acute renal failure. $91 \%$ of patients had hypertension syndrome. There were no maternal deaths. The average weight of newborns was 2,048 grams; $42 \%$ with gestational age less than 34 weeks; $34.4 \%$ weighed less than 1,500 grams; $9.4 \%$ had severe 5 minutes newborn depression; $12.1 \%$ died in the neonatal period. Conclusions: The HELLP syndrome is a disease of low incidence, with a high neonatal morbimortality.

KEY WORDS: HELLP syndrome, perinatal outcome, dexamethasone 


\section{INTRODUCCIÓN}

En 1982, Louis Weinstein $(1,2)$ acuñó el acrónimo en idioma inglés HELLP (Hemolysis, Elevated Liver Enzymes, Low Platelets) para referirse a una variante de la preeclampsia severa publicando una casuística de 29 pacientes con esta patología.

El síndrome HELLP es una patología grave del embarazo, con una mortalidad materna y perinatal de $4 \%$ (3) y de 73 por 1000 (4) respectivamente. Desde el punto de vista fisiopatológico se explica por una interacción anormal entre la microvasculatura de diferentes órganos y los elementos figurados de la sangre; que a nivel hepático se caracteriza por daño endotelial seguido de activación y agregación plaquetaria, que finalmente resulta en isquemia distal con muerte del hepatocito, esta vasculopatía puede estar limitada en un segmento hepático (necrosis periportal o focal) o difusamente a través de todo el hígado (infarto hepático y hematoma subcapsular) (5-8). Esto se traduce en alteraciones evidentes del laboratorio hematológico como trombocitopenia, anemia hemolítica microangiopática y liberación de productos celulares, particularmente a nivel hepático, incluyendo transaminasas y deshidrogenasa láctica. Se estima que el síndrome HELLP complica aproximadamente el $30 \%$ de los embarazos con preeclampsia severa o eclampsia, con una incidencia en EEUU de 1 por 1.000 embarazos (2).

El propósito de la presente investigación es conocer la incidencia general de síndrome HELLP en el Hospital Regional de Puerto Montt, con sus características epidemiológicas, clínicas, manejo médico y complicaciones materno-perinatales.

\section{PACIENTES Y MÉTODO}

Estudio de corte transversal. Se revisaron los egresos hospitalarios ocurridos en el servicio de Obstetricia y Ginecología del Hospital de Puerto Montt, entre el $1^{\circ}$ de enero de 2000 al 31 de diciembre de 2006. Se identificaron 33 pacientes con el diagnóstico de síndrome HELLP. El síndrome HELLP fue definido por los siguientes criterios: 1) Hemólisis: descenso del hematocrito y frotis sanguíneo característico; 2) Enzimas hepáticas elevadas: AST (GPT, GOT) >70 UI/L, LDH >600 UI/L; 3) Trombocitopenia: recuento plaquetario $<100.000$ por $\mathrm{mm}^{3}$. Se definió como tratamiento con dexametasona toda paciente que recibió el fármaco con fines terapéuticos en dosis intramuscular o endovenosa, pre o post parto. Las fichas clínicas fueron revisadas y la información obtenida fue ingresada a una base de datos, la que fue analizada con el programa estadístico STATA9.

\section{RESULTADOS}

Durante el período evaluado ocurrieron 25.226 partos. Se identificaron 33 pacientes con síndrome HELLP, con una de incidencia de 1,31 por 1000 partos para el periodo estudiado. El rango de las diferentes tasas por año fue de 0,28 a 1,99 por 1000 partos (Tabla I).

Las características generales de las pacientes

Tabla I

\section{INCIDENCIA (x 1000 partos) DE SÍNDROME HELLP: PUERTO MONTT 2000-2005}

\begin{tabular}{cccc}
\hline Año & $\mathrm{n}$ & Partos & Incidencia \\
\hline 2000 & 1 & 3.531 & 0,28 \\
2001 & 7 & 3.747 & 1,87 \\
2002 & 5 & 3.826 & 1,31 \\
2003 & 5 & 3.598 & 1,39 \\
2004 & 7 & 3.523 & 1,99 \\
2005 & 2 & 3.534 & 0,57 \\
2006 & 6 & 3.467 & 1,73 \\
Total & 33 & 25.226 & 1,31 \\
\hline
\end{tabular}

se presentan en la Tabla II. El promedio de edad de las pacientes fue $26,3 \pm 8,1$ años. El $39,4 \%$ de ellas era primigesta. El $29,7 \%$ de los casos era de procedencia rural. La talla materna promedio fue de $153,4 \pm 6,7 \mathrm{~cm}$. El promedio de edad gestacional de ingreso al control prenatal (CPN) fue de 15,3 \pm 8,1 semanas, con un promedio de asistencia de 5,7 $\pm 2,9$ veces. El peso materno promedio al ingreso del CPN fue de 60,7 $\pm 8,3$ kilos y al momento del último control de 72,4 $\pm 9,6$ kilos. El índice de masa corporal (IMC) al inicio del CPN fue de 26,8 $\pm 3,04$ $y$ al momento del parto de $31,1 \pm 3,5$. El $47 \%$ y el $19 \%$ de las pacientes se encontraban en sobrepeso y obesas respectivamente al inicio del embarazo según IMC.

Las características obstétricas de las pacientes se presentan en la Tabla III, destacándose a continuación las más relevantes. El $84,8 \%$ de las pacientes presentó HELLP preparto y $15,2 \%$ postparto. La presión arterial sistólica (PAS) promedio al ingreso fue $153,3 \pm 19,7 \mathrm{mmHg}$ y la presión arterial diastólica (PAD) de 97,2 $\pm 19 \mathrm{mmHg}$; el $41 \%$ de las pacientes presentó $P A S \geq 160 \mathrm{mmHg}$ y $24 \%$ PAD $\geq 110 \mathrm{mmHg}$; el $15,5 \%$ de las pacientes presentó 
presión arterial $\geq$ a 160/110 mmHg. El diagnóstico de ingreso fue en el $81,4 \%$ de las pacientes síndrome hipertensivo del embarazo, el $9,4 \%$ de eclampsia y el 6,3\% como síndrome HELLP. En el $96,7 \%$ de los casos la vía del parto fue cesárea. El 92,3\% de las pacientes con edad gestacional menor a 34 semanas tuvo maduración pulmonar fetal con betametasona. En 9 pacientes $(27,3 \%)$ se usó dexa-

\section{Tabla II}

CARACTERÍSTICAS GENERALES DE LAS MADRES CON SÍNDROME HELLP

\begin{tabular}{lr}
\hline Variable & Resultado \\
\hline Edad (años) & $26,3 \pm 8,1$ \\
$\begin{array}{l}\text { Paridad (\%): } \\
\text { • Primigesta }\end{array}$ & $13(39,4)$ \\
• Multípara 1 & $10(30,3)$ \\
• Multípara 2 & $8(24,2)$ \\
• Multípara 3 o más & $2(6,1)$
\end{tabular}

Procedencia:

- Urbana

- Rural

$29,7 \%$

Talla Materna $(\mathrm{cm})$

$153,4 \pm 6,7$

Peso materno $(\mathrm{kg})$

- Inicio control prenatal

- Al parto

$60,7 \pm 8,3$

$72,4 \pm 9,6$

Índice de masa corporal materna

- Inicio control prenatal

$26,8 \pm 3,04$

- Al parto

$31,1 \pm 3,5$

Estado nutricional al inicio del control prenatal, según IMC

- Normal (IMC 20-24,9)

- Sobrepeso (IMC 25-29,9)

- Obesidad (IMC 30 y más) metasona (8-10 mg ev cada 12 horas) para tratar el síndrome HELLP; 8 de ellas fueron tratadas preparto y 1 post parto con un número de 4 dosis en promedio (rango de 2 a 10 dosis). El parto ocurrió en promedio a las $34,2 \pm 4,4$ semanas.

Los valores de los exámenes de ingreso y control a las 48 horas post ingreso, se presentan en la Tabla IV.

\section{Tabla III}

\section{CARACTERÍSTICAS OBSTÉTRICAS DE LAS} MADRES CON SÍNDROME HELLP

Edad gestacional obstétrica $32,9 \pm 4$ a la hospitalización

Momento del diagnóstico

- Pre parto

- Post parto

Presión arterial $\geq 160 / 110$ mmHg

$15,5 \%$

Síntomas de ingreso

- Inespecíficos

$60,0 \%$

- Epigastralgia

- Cefalea

$N^{\circ}$ días de hospitalización

$3,8 \pm 4$ hasta el parto

Corticoides para maduración fetal $92,3 \%$ $<34$ semanas

Dexametasona para tratamiento HELLP

\section{$50 \%$}

HELLP $<34$ semanas

Edad gestacional al parto (semanas)

$34,2 \pm 4,4$

Vía del parto

- Vaginal 3\%

- Cesárea

Tipo de anestesia usada en el parto

- General

- Espinal

$75,8 \%$

- Peridural

- No consignado

Días de hospitalización desde 
Tabla IV

\section{CARACTERÍSTICAS DE LOS EXÁMENES DE LABORATORIO}

\begin{tabular}{lcc}
\hline \multicolumn{1}{c}{ Examen } & Ingreso & 48 horas control \\
\hline Hematocrito $(\%)$ & $35,7 \pm 5,1$ & $32,1 \pm 4,7$ \\
Hemoglobina $(\mathrm{g} / \mathrm{dl})$ & $11,6 \pm 1,5$ & $11,0 \pm 2,1$ \\
Plaquetas $\left(\mathrm{xmm}^{3}\right)$ & $102.830 \pm 53.979$ & $96.550 \pm 54.803$ \\
LDH (UI/L) & $608,1 \pm 1.142,9$ & $575 \pm 1.049,4$ \\
GOT (UI/L) & $209,9 \pm 408,6$ & $202,7 \pm 306,8$ \\
GPT(UI/L) & $86,9 \pm 123,0$ & $88,28 \pm 103,2$ \\
\hline
\end{tabular}

Las características generales de los recién nacidos (RN) se presentan en la Tabla V. El 41,9\% fueron menores de 34 semanas de gestación y el $29 \%$ fue de término. El peso promedio fue $2.048,8$ $\pm 947,5$ gramos, con $51,5 \%$ de sexo femenino. La frecuencia de fetos pequeños para la edad gestacional fue $54,8 \%$. El $34,4 \%$ fueron de muy bajo peso de nacimiento $(<1500 \mathrm{~g})$. El puntaje de Apgar promedio al minuto fue de $7,3 \pm 2,3$ y a los 5 minutos de $8,1 \pm 2,2 ; 42 \%$ de los RN necesitó reanimación neonatal. La depresión neonatal al minuto y cinco minutos de vida se observó en 18,8 y $9,4 \%$ respectivamente. Se registraron 3 casos de muerte neonatal precoz y 1 muerte fetal tardía.

Respecto a la evolución materna, el promedio de estadía hospitalaria hasta el alta fue de $9 \pm 4,7$ días. No se registraron muertes maternas. Siete pacientes $(21,2 \%)$ fueron hospitalizadas en la $\mathrm{UCl}$. El promedio de días de estada en $\mathrm{UCl}$ adulto fue de 3,5 días (Tabla III). La causa más importante de hospitalización materna en UCI fue la insuficiencia renal aguda, ninguna necesitó diálisis (Tabla VI).

\section{DISCUSIÓN}

El síndrome HELLP representa una patología de carácter grave, de alto riesgo de morbimortalidad materno-perinatal. Hasta el momento la fisiopatología de este cuadro no es del todo entendida. El estado del conocimiento actual nos indica que productos derivados de la placenta dañan los hepatocitos en el contexto de un síndrome inmunológico e inflamatorio agudo similar al síndrome de respuesta inflamatoria sistémica en el que el daño endotelial y la activación plaquetaria son el punto de convergencia de los mecanismos fisiopatológicos de la enfermedad (5-9).

La incidencia de esta patología en nuestra experiencia (1,31 por mil partos) es similar a la señalada en un estudio efectuado en la Región Metropolitana
Tabla V

\section{CARACTERÍSTICAS GENERALES DE LOS RECIÉN NACIDOS DE MADRES CON SÍNDROME HELLP}

\begin{tabular}{lc}
\hline Variable & Resultado \\
\hline Peso recién nacido (g) & $2.048 \pm 947,5$ \\
Relación peso talla (\%) & \\
$\bullet$ PEG & 54,8 \\
$\cdot$ AEG & 41,9 \\
$\bullet$ GEG & 3,2 \\
Recién nacido < 1.500 g & $34,4 \%$ \\
Recién nacido <34 s & $41,9 \%$ \\
Apgar al minuto (\%) & \\
$\bullet 0-3$ & $3(9,4)$ \\
$\bullet 4-6$ & $3(9,4)$ \\
$\bullet 7-10$ & $26(81,3)$ \\
Apgar al $5^{\circ}$ minuto (\%) & \\
$\bullet 0-3$ & $2(6,3)$ \\
$\bullet 4-6$ & $1(3,1)$ \\
$\bullet 7-10$ & $29(90,6)$ \\
Hospitalización UCI RN & 6 \\
\hline
\end{tabular}

Tabla VI

PRINCIPALES CAUSAS DE HOSPITALIZACIÓN EN UCI DE MADRES CON SÍNDROME HELLP

\begin{tabular}{lr}
\hline Patologia & $\mathrm{n}$ \\
Insuficiencia renal aguda & 3 \\
Hematoma a herida operatoria & 2 \\
Hemorragia primaria post parto & 1 \\
Insuficiencia respiratoria aguda & 1 \\
Emergencia hipertensiva & 2 \\
Sospecha de hematoma & 1 \\
subcapsular hepático &
\end{tabular}

\section{(1,53 por mil partos) (10).}

En nuestro estudio podemos constatar algunos factores de riesgo señalados en la literatura, entre ellos: la multiparidad $(60,6 \%$ de las pacientes) y la edad materna sobre 25 años (edad promedio: 26 años y $57,6 \%$ tenía 25 años o más) $(10,11)$. En nuestra serie un $47,6 \%$ y $19,1 \%$ de las pacientes estaban en sobrepeso y obesas, respectivamente, al inicio del embarazo de acuerdo a la gráfica de Atalah y cols para evaluación nutricional de la 
embarazada según índice de masa corporal (12), siendo el sobrepeso y la obesidad pregestacional factores de riesgo de preeclampsia, diabetes gestacional, óbito fetal y malformaciones del sistema nervioso central fetal entre otros $(13,14)$. En Chile la prevalencia de obesidad en mujeres embarazadas ha aumentado de $15 \%$ a $32 \%$ en el periodo 1987-2000 (15), de tal forma que la actividades de promoción en salud con educación nutricional y de actividad física para mantener un peso normal y saludable son importantes antes del embarazo. He aquí un desafío importante para la salud pública en área de la salud materna perinatal.

Los signos y síntomas descritos en la literatura para el síndrome HELLP son variables, se puede o no asociar a hipertensión, cefalea (33\%-68\%), malestar general $(90 \%)$, dolor epigástrico o en el cuadrante superior derecho del abdomen (33$90 \%$ ), náuseas y vómitos (11). En nuestro estudio la frecuencia de presentación es distinta ya que sólo $15,2 \%$ tiene consignado en la ficha clínica la presencia de epigastralgia y $9 \%$ de cefalea al momento de ingreso, por lo que debe existir un importante subregistro de esos importantes síntomas. El malestar general o inespecífico fue lo más frecuente $(60 \%)$, y $13,8 \%$ de las pacientes presentaron una PA menor de 140/90 mmHg. Las diferencias dependen del estado de gravedad de la enfermedad y de la anamnesis dirigida ante la sospecha diagnóstica. Se ha señalado que el dolor epigástrico en las pacientes con HELLP tiene parámetros de laboratorio más alterados y más días de hospitalización sin aumento de complicaciones maternas (16).

Se debe tener presente que toda paciente con dolor epigástrico o en el cuadrante superior derecho del abdomen durante la segunda mitad del embarazo puede estar cursando con un síndrome HELLP, más aun toda embarazada con signos y síntomas de preeclampsia severa que presenta dolor abdominal epigástrico intenso o en el cuadrante superior derecho abdominal, puede estar presentando hemorragia con hematoma subcapsular hepático o ruptura hepática. (9). La asociación con el síndrome hipertensivo del embrazo es conocida, el $95,8 \%$ de las pacientes con HELLP fue hospitalizada por esta causa, de hecho el HELLP se presenta entre el 4 a $12 \%$ de las pacientes con preeclampsia-eclampsia (16).

El manejo del síndrome HELLP, luego de la estabilización hemodinámica de la paciente (hipotensores y aporte de volumen), es la interrupción del embarazo; removiendo la placenta y el tejido decidual (17). En nuestro centro no tenemos una conducta expectante una vez diagnosticado el cuadro, con excepción del tiempo necesario para la inducción de madurez pulmonar. Se ha descrito que la gravedad de esta patología puede disminuir con el uso de corticoides. Las primeras evidencias surgen de 2 pequeños trabajos randomizados que utilizaron altas dosis de dexametasona versus no uso de corticoides; estos trabajos mostraron estabilización y mejoría significativa en los parámetros clínicos y de laboratorio en las pacientes que recibieron corticoides $(18,19)$. La evidencia actual es controversial, Fonseca y cols (20) publican un estudio randomizado de 132 mujeres con HELLP en que el tratamiento con dexametasona no fue más eficaz que el placebo, pero su estudio evalúa variables poco relevantes como duración de la hospitalización en una población heterogénea con bajas dosis de corticoides (10 mg ev cada 12 horas hasta el parto y 3 dosis adicionales en el post parto entre 3 a $10 \mathrm{mg}$ ev) (21-23). Dos metaanálisis que evalúan la evidencia de los efectos de los corticoides en la morbimortalidad materna y neonatal, concluyen que no hay evidencia suficiente para sostener que el tratamiento con dexametasona o betametasona pueda ser usado como el tratamiento estándar del síndrome HELLP $(24,25)$.

En Chile no existen datos publicados sobre los beneficios de la dexametasona en esta patología. Encontramos un reporte de caso clínico en el que su uso fue beneficioso basado en la evolución clínica y de laboratorio (26). En nuestra serie, 27\% de las pacientes recibió dexametasona como tratamiento del síndrome HELLP, esto se explica por la baja sospecha diagnóstica inicial en las pacientes $(6,3 \%)$ y porque no teníamos un protocolo de manejo. Respecto a la evolución clínica de nuestras pacientes con el uso de dexametasona no podemos ofrecer conclusiones ya que las dosis y la duración del tratamiento fueron heterogéneas. En un estudio que evaluó la práctica clínica de 298 obstetras en 7 temas controversiales, entre ellos el síndrome HELLP, se observó que el $50 \%$ y el $30 \%$ de los evaluados usa altas dosis de corticoides para tratar el preparto y postparto respectivamente, a pesar de no estar demostrada su utilidad (27). Urge, por lo tanto, tener un estudio clínico nacional multicéntrico aleatorizado sobre la utilidad de la dexametasona en altas dosis en esta patología como las sugeridas por O’Brien y cols (28). En nuestro hospital instauraremos un protocolo de seguimiento del manejo y resultados con dosis altas de dexametasona una vez diagnosticado el síndrome HELLP.

El 21,2\% de las pacientes se hospitalizó en UCI, las complicaciones observadas fueron escasas; la más frecuente fue la insuficiencia renal aguda (3 casos), que según la literatura se presenta en el $20 \%$ a $53,8 \%$ de los casos $(29,30)$ y de los cuales 
33 a $86 \%(29,31)$ puede llegar a necesitar diálisis pudiendo llegar a una mortalidad materna entre 12 y $17,1 \%$ (29). Las causas obstétricas dan cuenta del $12,4 \%$ de las causas de insuficiencia renal aguda y de éstas, el síndrome HELLP corresponde entre 35 a $45,7 \%(31,32)$. No se registraron otros tipos de complicaciones (pulmonares, neurológicas ni hematológicas). Tampoco tuvimos complicaciones hepáticas, a diferencia de una serie nacional reciente de 32 pacientes, en que Almuna y cols reportan 3 casos de hematoma con rotura hepática sin casos fatales (10). La rotura hepática es una complicación extremamente grave del HELLP con una incidencia reportada en Santiago de Chile de 1 en 28.597 partos en el Hospital Barros Luco Trudeau (33) a 1 en 7.879 en el Hospital Dr. Luis Tisné Brousse (10); y en series extranjeras de1:45.000 a 1:225.000 partos. La rotura hepática ocurre en un 2 a $4 \%$ de las pacientes con síndrome HELLP con una mortalidad materna de $18-86 \%$ (34-36) y una mortalidad perinatal de $80 \%$. Dadas estas características, su manejo implica una cooperación interdisciplinaria entre obstetra, intensivista y cirujano con un concepto agresivo de tratamiento quirúrgico (packing, ligadura arteria hepática, transplante hepático) y de cuidados intensivos (35-37). Se destaca que en nuestra serie no hubo muertes maternas.

En cuanto a los resultados neonatales, las pacientes con síndrome HELLP presentan elevadas tasas de prematurez ( $42 \%$ menores de 34 semanas), de recién nacidos de muy bajo peso al nacer (34\%), depresión neonatal severa y alta mortalidad neonatal $(21 \%)$.

\section{CONCLUSIÓN}

Nuestro centro presenta una incidencia de síndrome HELLP similar a la reportada a nivel nacional, y a pesar de ser una entidad poco frecuente, repercute en forma importante en la morbimortalidad materno-perinatal tal como se describe en la literatura. La sospecha clínica fue baja, condición que podría empeorar aún más el resultado perinatal. Se debiera implementar un protocolo nacional para la administración de dexametasona y homogenizar criterios para el manejo de estas pacientes.

\section{BIBLIOGRAFÍA}

1. Weinstein L. Syndrome of hemolysis, elevated liver enzymes, and low platelet count: a severe consequence of hypertension in pregnancy. Am J Obstet Gynecol 1982;142(2):159-67.

2. Weinstein L. It has been a great ride: The history of HELLP syndrome Am J Obstet Gynecol 2005;193:860-3.

3. Isler CM, Rinehart BK, Terrone DA, Martin RW, Magann EF, Martin JN Jr. Maternal mortality associa- ted with HELLP syndrome. Am J Obstet Gynecol 1999;181(4):924-8.

4. Martin JN Jr, Rinehart BK, May WL, Magann EF, Terrone DA, Blake PG. The spectrum of severe preeclampsia: comparative analysis by HELLP. Am J Obstet Gynecol 1999;180:1373-84.

5. Stratta P, Canavese C, Vercellone A. HELLP, microangiopathic hemolytic anemia, and preeclampsia. Hyperten Pregnancy 1993;12:487-96.

6. Roberts JM, Cooper DW. Pathogenesis and genetics of pre-eclampsia. Lancet 2001;357(9249):53-6.

7. Roberts JM. Preeclampsia, what we know and what we do not know. Semin Perinatol 2000;24(1):24-8.

8. Martin JN Jr, Rose $\mathrm{CH}$, Briery $\mathrm{CN}$. Understanding and managing HELLP syndrome: The integral role of aggressive glucocorticoids for mother and child. Am J Obstet Gynecol 2006; 195(4): 914-34.

9. Strand S, Strand D, Seufert R, et al. Placenta-Derived CD95 ligand causes liver damage in hemolysis, elevated liver enzymes, and low platelet count syndrome. Gastroenterology 2004;126(3): 849-58.

10. Almuna R, Vera D, Aedo S, et al. Antecedentes y complicaciones en 32 pacientes con síndrome HELLP. Rev Obstet Ginecol Hosp Santiago Oriente Dr. Luis Tisné Brousse 2007;2(1):23-8.

11. Sibai BM, Ramadan MK, et al. Maternal morbidity and mortality in 442 pregnancies with hemolysis, elevated liver enzymes, and low platelets (HELLP syndrome). Am J Obstet Gynecol 1993;169(4):1000-6.

12. Atalah E, Castillo C, Castro R, Aldea A. Propuesta de un nuevo estándar de evaluación nutricional en embarazadas. Rev Méd Chile 1997;125(12):1429-36.

13. Atalah E, Castro R. Obesidad materna y riesgo reproductivo. Rev Méd Chile 2004; 132: 923-930.

14. O'Brien TE, Ray JG, Chan WS. Maternal body mass index and the risk of preeclampsia: a systematic overview. Epidemiology 2003;14(3):368-74.

15. Mardones F. Evolución de la antropometría materna y del peso de nacimiento en Chile, 1987-2000. Rev Chil Nutr 2003;30:122-31.

16. Moore L, Martin JN, Thigpen B, Rose C, Cushman J, May W. Implications of epigastric pain in HELLP syndrome. Obstet Gynecol 2003; 101, S 64.

17. Magaan EF, Martin JN Jr. Critical care of HELLP Syndrome with corticosteroids. Am J Perinatol 2000; 17(8): 417-22.

18. Magann EF, Bass D, Chauhan SP, et al. Antepartum corticosteroids: disease stabilization in patients with the syndrome of hemolysis, elevated liver enzymes, and low platelets (HELLP). Am J Obstet Gynecol 1994;171(4):1148-53.

19. Magann EF, Perry KG Jr, Meydrech EF, et al. Postpartum corticosteroids: accelerated recovery from HELLP syndrome. Am J Obstet Gynecol 1994;171(4):1154-8.

20. Fonseca JE, Méndez F, Cataño C, Arias F. Dexamethasone treatment does not improve the outcome of women with HELLP syndrome: A double-blind, placebo-controlled, randomized clinical trial. Am J Obstet Gynecol 2005;193(5):1591-8.

21. O'Brien JM. Dexamethasone, HELLP syndrome, and study design. Am J Obstet Gynecol 2006;195(6);134.

22. Mould S, Paruk F, Moodley J. High-dose dexametha- 
sone in the treatment of HELLP syndrome. Int J Gynecol Obstet 2006;93(2):140-1.

23. Sibai BM. Diagnosis, controversies, and management of the syndrome of hemolysis, elevated liver enzymes, and low platelet count. Obstet Gynecol 2004;103:98191.

24. Matchaba P, Moodley J. Corticosteroids for HELLP syndrome in pregnancy. The Cochrane Database of Systematic Reviews 2004, Issue1. Art. No.: CD002076.pub2. DOI: 10.1002/14651858.CD002076. pub2.

25. Vidaeff AC, Yeomans ER. Corticosteroids for the syndrome of hemolysis, elevated liver enzymes, and low platelets (HELLP): what evidence? Minerva Ginecol 2007;59(2):183-90.

26. Almuna R, Valdés L, Ramirez C. Eficacia de la dexametasona en síndrome HELLP. Rev Chil Obstet Ginecol 2003:68;508-12.

27. Norwitz E, Bahtiyar M, Sibai B. Defining standards of care in maternal-fetal medicine. Am J Obstet Gynecol 2004;191(4):1491-6.

28. O'Brien JM, Milligan DA, Barton JR. Impact of highdose corticosteroid therapy for patients with HELLP (hemolysis, elevated liver enzymes, and low platelet count) syndrome. Am J Obstet Gynecol. 2000;183(4):921-4.

29. Martínez de Ita AL, García Cáceres E, Helguera Martínez AM, Cejudo Carranza E. Acute renal insufficiency in HELLP syndrome. Ginecol Obstet Mex
1998;66:462-8.

30. Abraham KA, Connolly G, Farrell J, Walshe JJ. The HELLP syndrome, a prospective study. Ren Fail 2001;23(5):705-13.

31. Selçuk NY, Odabas AR, Cetinkaya R, Tonbul HZ, San A. Outcome of pregnancies with HELLP syndrome complicated by acute renal failure (1989-1999). Ren Fail 2000;22(3):319-27.

32. Altintepe L, Guney I, Tonbul Z, Demir M, Turk S, Yeksan M. Assessment of acute renal failure patients treated in our nephrology clinic between 1996 and 2002. Transplant Proc 2004;36(10):3002-5.

33. Kuczkowski KM. Hepatic manifestations and complications of HELLP syndrome. Ann Fr Anesth Reanim 2006; 26(3): 264-5.

34. Perucca E, Domínguez C, González D, et al. Rotura hepática espontánea en síndrome HELLP. Rev Chil Obstet Ginecol 2003;68:7-12.

35. Reck T, Bussenius-Kammerer M, Ott R, et al. Surgical treatment of HELLP syndrome -associated liver rupture- an update. Eur J Obstet Gynecol Reprod Biol 2001;99(1):57-65.

36. Araujo AC, Leao MD, Nobrega MH, et al. Characteristics and treatment of hepatic rupture caused by HELLP syndrome. Am J Obstet Gynecol 2006;195(1):12933.

37. Tank PD, Nadanwar YS, Mayadeo NM. Outcome of pregnancy with severe liver disease. Int $\mathrm{J}$ Gynecol Obstet 2002;76(1):27-31. 